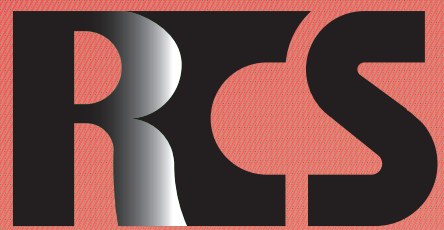

Depósito legal ppi $201502 Z U 4662$

Esta publicación científica en formato digital es continuidad de la revista impresa Depósito Legal: pp $197402 Z U 789$

- ISSN: 1315-9518 • ISSN-E: 2477-9431

Revista de Ciencias Sociales

Universidad del Zulia. Revista de la Facultad de Ciencias Económicas y Sociales Vol. XXVII. No. 2

Abril-Junio 2021

Esta publicación científica en formato digital es continuidad de la revista impresa Depósito Legal: pp $197402 Z$ Z789 ISSN: 1315-9518 


\title{
Potencial paisajístico de la Laguna de Coyuca de Benítez: Detonante de productos sustentables en Acapulco-México
}

\author{
Anzaldúa-Soulé, Karla Rosalba* \\ Almazán-Adame, Arely Adriana ${ }^{* *}$ \\ Lorenzana Núñez, Oswaldo ${ }^{* * *}$ \\ Saldaña Almazán, Mirella***
}

\section{Resumen}

El destino turístico Acapulco privilegió por décadas el desarrollo turístico tradicional, impulsando al turismo de masas, provocando transformación y modificación de escenarios naturales. El objetivo fue evaluar el paisaje de la Laguna de Coyuca de Benítez con potencial turístico para generar productos sustentables que permitan diversificar la oferta de Acapulco (México). Se consideró el enfoque mixto de valoración directa e indirecta, considerando cuatro fases: Las características socio-económicas y ambientales; se aplicó talleres participativos; fichas de observación; se definió las unidades de paisaje con base en el valor intrínseco y extrínseco. Los resultados arrojaron cinco unidades de paisaje con potencial turístico, clasificadas en paisaje rural, natural y urbano: Paisaje rural, se integró el Estero; como natural, la Isla la pelona y la zona de manglares; y, urbano, la Barra y la Isla Montosa; aun cuando esta isla todavía cuenta con una amplia extensión territorial sin modificaciones por el hombre, es un área que constantemente se encuentra aprovechada por las actividades turísticas. Concluyendo, que para lograr que las zonas identificadas sean soportables ecológicamente a largo plazo, viables económicamente y equitativas desde una perspectiva ética y social, será indispensable impulsar la participación de las comunidades y la aplicación de políticas de protección ambiental.

Palabras clave: Paisaje; aprovechamiento sustentable; desarrollo turístico; participación comunitaria; Acapulco-México.

\footnotetext{
* Doctora en Ciencias Ambientales. Maestra en Administración e Innovación del Turismo. Profesora Investigadora de la Unidad Académica de Turismo en la Universidad Autónoma de Guerrero, Acapulco-México. E-mail: karlasoule88@ gmail.com (iD ORCID: https://orcid.org/0000-0002-2161-842X

** Doctora. Profesora Investigadora de la Unidad Académica de Ciencias Económicas en la Universidad Autónoma de Guerrero, Acapulco-México. E-mail: arelyalmazan@hotmail.com iD ORCID: https://orcid.org/0000-0002-61055748

*** Maestro en Administración e Innovación del Turismo. Profesor Investigador de la Unidad Académica de Turismo en la Universidad Autónoma de Guerrero, Acapulco-México. E-mail: oswaldoln@hotmail.com iD ORCID: https:// orcid.org/0000-0001-9145-8970

**** Doctora en Ciencias Ambientales. Magister en Administración. Profesora Investigadora del Centro de Ciencias de Desarrollo Regional en la Universidad Autónoma de Guerrero, Acapulco-México. E-mail: mirellasal@hotmail.com (iD) ORCID: $\underline{\text { https://orcid.org/0000-0001-8995-1803 }}$
} 


\title{
Landscape potential of the Coyuca de Benítez Lagoon: Trigger for sustainable products in Acapulco-Mexico
}

\begin{abstract}
The Acapulco tourist destination privileged traditional tourism development for decades, promoting mass tourism, causing transformation and modification of natural settings. The objective was to evaluate the landscape of the Coyuca de Benítez Lagoon with tourism potential to generate sustainable products that allow diversifying the offer of Acapulco (Mexico). The mixed approach of direct and indirect valuation was considered, considering four phases: The socio-economic and environmental characteristics; participatory workshops were applied; observation cards; Landscape units were defined based on intrinsic and extrinsic value. The results yielded five landscape units with tourist potential, classified as rural, natural and urban landscapes: Rural landscape, the Estero was integrated; as natural, the Isla la pelona and the mangrove area; and, urban, Barra and Isla Montosa; Even though this island still has a wide territorial extension without modifications by man, it is an area that is constantly used by tourist activities. Concluding, that in order to make the identified areas ecologically bearable in the long term, economically viable and equitable from an ethical and social perspective, it will be essential to promote the participation of communities and the application of environmental protection policies.
\end{abstract}

Keywords: Landscape; sustainable use; tourism development; community participation; Acapulco-Mexico.

\section{Introducción}

El paisaje es un recurso de alto valor, que integra elementos naturales y sociales que marcan la identidad de un destino turístico. El identificar elementos potenciales del paisaje en zonas sin aprovechamiento, pueden contribuir para la integración de nuevos productos turísticos en destinos consolidados, que necesiten fincar el desarrollo del turismo dentro del marco de la sustentabilidad. Al respecto, "se puede evidenciar cada día el interés de parte de los Estados en impulsar proyectos viables, innovadores y de fortalecimiento en pro de la estabilidad y bienestar de la población" (Guillen, et al, 2020, p.294) en los entornos económico, social, así como ambiental.

Tal es el caso, de Acapulco-México que a mitad del siglo XX el puerto se consolidó como el principal destino turístico del país (Alcaraz y Salgado, 2019); sin embargo, en el transcurso de los años fue perdiendo competitividad, por lo tanto, es necesario descubrir nuevas zonas que permitan impulsar una visión de desarrollo diferente, que busque el equilibrio entre lo ambiental, económico y social. En este sentido, la investigación propuso evaluar la potencialidad del paisaje de La Laguna de Coyuca de Benítez, con el fin de integrar productos turísticos sustentables que permitan diversificar la oferta de Acapulco.

El objeto de estudio se localiza compartiendo su territorio con los municipios de Acapulco de Juárez y Coyuca de Benítez del Estado de Guerrero, es un ecosistema de manglar que mide diez kilómetros de este a oeste, con una anchura de más de cinco kilómetros y desemboca al oeste por un canal, en la boca del río del mismo nombre. En ella habitan reptiles, aves, peces, además alberga dos islas: Isla Montosa e Isla Presidio, que pertenecen al Municipio de Coyuca de Benítez (Márquez-García, Campos-Verduzco y Castro-Soriano, 2010; Instituto Nacional de Estadística y Geografía [INEGI], 2015a).

La metodología planteó cuatro fases, permitiendo realizar un diagnóstico socioeconómico y ambiental; la aplicación de 
Anzaldúa-Soulé, Karla Rosalba; Almazán-Adame, Arely Adriana; Lorenzana Núñez, Oswaldo y Saldaña Almazán, Mirella

Potencial paisajistico de la Laguna de Coyuca de Benitez: Detonante de productos sustentables en Acapulco-México

talleres participativos, tal garantía descansa en el supuesto de que las comunidades que tienen un fuerte sentido de su identidad y su propio valor cultural, patrimonial y estilo de vida, están en una buena posición para realizar un aprovechamiento del turismo formado por su propio sentido del valor de la comunidad e integrando un atractivo para los potenciales turistas (Macbeth, Carson y Northcote, 2004; Ordoñez y Ochoa, 2020); la aplicación de fichas de observación, para homogeneizar el territorio en unidades de paisaje; y, la adaptación así como aplicación (Van den Berg y Van Winsum-Westra, 2010) del método mixto de valoración directa e indirecta de Alberruche (2002); y, Delgado y Pantoja (2016).

Finalmente, es importante considerar que el logro del aprovechamiento sustentable del paisaje en un destino turístico, dependerá de la gestión pública y privada, por medio de políticas así como estrategias que permitan marcar los límites de uso y ordenamiento, con la finalidad de lograr la protección de los recursos naturales y sociales.

\section{Fundamentación teórica}

\subsection{La potencialidad y desarrollo turístico sustentable del paisaje}

El paisaje, tiene un importante papel en el interés público en aspectos culturales, sociales, ambientales, y constituye un recurso favorable para la actividad económica; es el elemento clave de la gestión social para la comprensión de las características históricas y evolutivas de la realidad en un área particular (Maloguelonne Déjeant-Pons Head of the Spatial Planning and Landscape Division, 2006). No solo resulta un concepto aparentemente accesible y comprensible para amplios sectores de la sociedad, sino que además tiene un fuerte componente identitario colectivo y sensibilización con las transformaciones del territorio (Méndez, et al., 2016).

En ese sentido, el paisaje es analizado a partir de diversas visiones, desde una perspectiva turística, representa uno de los principales pilares; es aprovechado como una imagen, con el fin de difundir una determinada región o centro turístico (Nogué, 1992). Además, brinda activos y límites para el desarrollo turístico e indirectamente proporciona el marco en el que a menudo se concibe el turismo como una herramienta de desarrollo regional (Stoffelen y Vanneste, 2015; Méndez-Méndez, et al., 2018).

Asimismo, el turismo ha logrado aprovechar el conjunto de elementos que integran el paisaje como son: Los elementos naturales y culturales, mismos que determinan la identidad y lo convirtieren en un elemento substancial del fenómeno turístico así como en un recurso de gran valor para el desarrollo y la consolidación de la oferta turística (Maguelonne Déjeant-Pons Head of the Spatial Planning and Landscape Division, 2006; Włodarczyk, 2009; Dos Santos, 2011).

Existen zonas con paisajes que poseen potencial natural y cultural, el cual, se define por la capacidad de las características y propiedades naturales de un área o zona determinada que permiten o favorecen su utilización con el fin de ser aprovechado para funciones socioeconómicas específicas, con el objeto de satisfacer las necesidades de la sociedad. De acuerdo con Pelegrín, et al. (2020), "no sólo las características físicas del paisaje, los elementos ambientales y culturales, sino también la evolución histórica, intervienen de manera decisiva y sirven de referente diferenciador a la hora de establecer la región turística" (p.232).

En ese sentido, las zonas con potencialidad para ofrecer un producto turístico deben vincular a los diferentes agentes sociales (entes gubernamentales, empresarios, sociedad civil, instituciones educativas), en los procesos de planificación de las dimensiones de desarrollo antes descritas (económica, social y ambiental), integrando para ello a todos los factores del sistema turístico con el fin de optimizar los recursos naturales, históricos y culturales de los cuales disponen (Narváez, Fernández y Gutiérrez, 2018).

Las evaluaciones actuales del paisaje, se 
basan generalmente en puntajes otorgados por expertos, que pueden ser juicios subjetivos o bien vinculados a las instalaciones turísticas (Burkhard, et al., 2009; Weyland y Laterra, 2014). Aunque la puntuación puede ser eficiente, existen limitaciones que influyen en la subjetividad, la imprecisión y la simplificación excesiva (Boerema, et al., 2017). Por lo tanto, las evaluaciones turísticas de paisaje tienden a ser muy superficiales, puesto que solo consideran el comportamiento de los turistas (Wozniak, Kulczyk y Derek, 2017).

Analizando diversas metodologías de evaluación del paisaje el estudio plantea comprender y valorar la percepción de la comunidad, así como las relaciones físicas y psicológicas de los usuarios con el territorio (Bramwell y Lane, 1993; Van den Berg y Van Winsum-Westra, 2010).

De acuerdo con lo anterior, se puede observar que existen investigaciones sobre el análisis del potencial paisajístico, que consideran por una parte la opinión de la comunidad, o la de un grupo de expertos; en este caso, la investigación pondera el indagar ambas aristas, con el objeto de conocer el panorama ambiental y social de la comunidad y someterlo al análisis de expertos.

En este mismo sentido, el paisaje se convierte en un elemento de gran valor para consolidarse como parte de una oferta turística, sin dejar de señalar que es un recurso delicado porque se deteriora o se altera y su recuperación es compleja (Suárez, 2014). Por lo tanto, el desarrollo turístico del paisaje tiene que ser considerado desde la visión del desarrollo sustentable, con el fin de no privilegiar el aprovechamiento irracional, que en Acapulco a permeado por décadas, puesto que, la:

Industria turística es promovida por los organismos internacionales, que buscan el desarrollo en los países emergentes, un turismo de masas que demanda muchos espacios, infraestructura y servicios. Esto ha transformado y modificado los escenarios naturales en pro del desarrollo sustentable, con los beneficios económicos de las grandes corporaciones multinacionales y con las consecuencias de deterioro en las regiones donde se implantan, dejando a las comunidades locales más problemas que beneficios pues los crecimientos provocados por la actividad se dan a un ritmo acelerado para satisfacer las necesidades de infraestructura y servicios que demanda el turismo. (Aguilar, Palafox y Anaya, 2015, p.21)

\section{Metodología}

La zona de estudio se situó en la Laguna de Coyuca de Benítez, localizada en el Estado de Guerrero, México, territorio que comparte su extensión con los municipios: Coyuca de Benítez y Acapulco de Juárez. El desarrollo de la investigación se enmarca en un enfoque mixto de valoración directa e indirecta, permitiendo combinar las ventajas inherentes de ambos métodos y evitar el sesgo de información (Hernández, Fernández y Baptista, 2014): Reconociendo la interpretación de la realidad del paisaje en su contexto socioeconómico y ambiental, así como, se determinó la calidad paisajística que alberga la zona para aprovecharse turísticamente con actividades que promuevan la conservación de sus recursos naturales. Así, a partir del análisis de diversas metodologías se estructuró el siguiente proceso integrado por cuatro fases:

a. En la primera fase: Se realizó el diagnóstico socioeconómico y ambiental del paisaje. Para este proceso se aplicó la investigación documental, permitiendo analizar diversas fuentes de información: Plataformas gubernamentales, artículos científicos, tesis, y libros. Los elementos que estructuraron las condiciones socioeconómicas son: Áreas de poblaciones urbanas, semi-urbanas y rurales; número de población total; población indígena; número de viviendas; índice de desarrollo social; grado de marginación; población ocupada y desocupada; así como, principales actividades económicas. Los aspectos ambientales que se obtuvieron son: Características del suelo, 
fisiografía, geología, hidrología, humedales, uso de suelo y vegetación.

b. En la segunda fase: Se ejecutó la investigación descriptiva, comenzando con la aplicación de dos talleres de participación comunitaria enfocados en actores clave (líderes naturales, sacerdotes, representantes de asociaciones y grupos, comisarios ejidales y municipales) para la valoración de los recursos tangibles e intangibles como: Naturales-ecológicos, históricos, estéticos, sociales, productivos, espirituales, simbólicos e identitarios (Nogué y Sala, 2008).

Los talleres estuvieron estructurados en tres apartados: El primero, fue la selección de los paisajes que consideran más originales y atractivos de la Laguna de Coyuca de Benítez, los cuales se jerarquizaron de acuerdo al orden de importancia donde uno es el de mayor valoración y diez el de menor. En el segundo apartado, la población seleccionó los paisajes de los cuales se sienten orgullosos y valoraron los siguientes elementos: Componentes del paisaje; valores; estado en que se encuentran (excelente, regular y malo); personas involucradas en la protección, gestión y ordenación; negocios, actividad o producto relacionado; nombre y fecha de la fiesta o evento que se celebra en el sitio. En el tercer apartado, se analizó el presente y fututo de los paisajes seleccionados, estudiando los siguientes elementos: Situación actual, amenazas, propuestas y visión deseada para el futuro.

Con la aplicación de los talleres se logra el desarrollo participativo que proporciona a los miembros de la comunidad la posibilidad de determinar qué tipo de acciones necesitan y como y cuando desarrollarlas (Organización de las Naciones Unidas para la Alimentación y la Agricultura [FAO], 2008). Finalmente, se concibe que ante cualquier planificación coherente de desarrollo turístico de un área geográfica, es indispensable considerar los diversos grupos de interés especialmente a la comunidad local, así como, sus percepciones y actitudes; con respecto a su desarrollo sociocultural, económico, ambiental (Núñez, Fuentes y Sánchez, 2014; Guillen, et al, 2020).

c. En la tercera fase: Se levantaron las fichas de observación como se puede observar en el Cuadro 1, que permitieron reconocer la ubicación geográfica y los elementos del paisaje: 1) Descripción (denominación) del paisaje; 2) valoración del paisaje (se ponderó de acuerdo al número de variables seleccionadas); 3 ) estado de conservación (se valoró con la escala de Likert); 4) grado de vulnerabilidad (selección directa de acuerdo a las variables). En lo que respecta, al elemento 5) la valoración patrimonial intrínseco del paisaje desde el punto de vista turístico, valoración de la contribución a la conservación y uso racional; se ponderó en función de un índice creciente del 1 al 5 en cada apartado, donde uno es el de menor y cinco el de mayor puntaje (Anzaldúa-Soulé, 2013). Otros elementos también considerados fueron: 6) Atracción, 7) accesibilidad, 8) lugares de equipamiento y 9) otros. 


\section{Cuadro 1 \\ Elementos de la ficha de observación}

\begin{tabular}{|c|c|c|c|}
\hline $\begin{array}{l}\text { 1. Denominación del } \\
\text { paisaje }\end{array}$ & $\begin{array}{l}\text { 3. Estado de } \\
\text { conservación }\end{array}$ & $\begin{array}{l}\text { 5. Valor patrimonial } \\
\text { intrínseco del paisaje desde el } \\
\text { Punto de vista turístico }\end{array}$ & 7. Accesibilidad \\
\hline a. Paisaje vestigios & a. Desaparecido & a. Significatividad & $\begin{array}{c}\text { a. Comunicaciones } \\
\text { viales }\end{array}$ \\
\hline b. Paisaje activo & b. Casi desaparecido & b. Representatividad & b. Equipamiento \\
\hline 2. Valoración del paisaje & c. Seriamente alterado & c. Singularidad. & $\begin{array}{l}\text { c. Instalaciones para } \\
\text { interpretación }\end{array}$ \\
\hline a. Estéticos & d. Poco alterado & 6. Atractividad & $\begin{array}{l}\text { 8. Lugares de } \\
\text { equipamiento }\end{array}$ \\
\hline b. Naturales y Ecológicos & $\begin{array}{l}\text { e. Paisaje activo bien } \\
\text { conservado }\end{array}$ & a. Belleza & a. Hoteles \\
\hline c. Productivos & $\begin{array}{c}\text { 4. Grado de } \\
\text { vulnerabilidad }\end{array}$ & b. Originalidad & b. Restaurantes \\
\hline d. Históricos & a. Sin protección física & c. Simbolismo & c. Atracciones \\
\hline e. Religiosos y espirituales & b. Difícil acceso & d. Emociones que provoca & d. Tours \\
\hline f. Uso social & $\begin{array}{c}\text { c. Área de protección } \\
\text { física }\end{array}$ & & e. Recorridos \\
\hline $\begin{array}{l}\text { g. Simbólicos e } \\
\text { identitarios }\end{array}$ & & & f. Renta de lanchas \\
\hline 9. Otros & & & \\
\hline
\end{tabular}

Fuente: Elaboración propia, 2020, a partir de Anzaldúa-Soulé (2013).

De acuerdo a los resultados del diagnóstico socioeconómico y ambiental, los talleres participativos y las fichas de observación, se homogeneizó el territorio de la Laguna de Coyuca de Benítez para la identificación de las unidades de paisaje, considerando seis variables: Factores fisiográficos; usos y las cubiertas del suelo; dimensión histórica del paisaje; estructura del paisaje; visibilidad; dinámicas recientes $\mathrm{y}$ tendencias inmediatas; $\mathrm{y}$, sentimientos del lugar (Nogué y Sala, 2008).

d. En la cuarta fase: Se aplicó la evaluación del paisaje con potencial turístico, realizando una adaptación con las propuestas de Alberruche (2002); y, Delgado y Pantoja (2016). Por lo tanto, se describen los elementos que son base para este proceso: El valor paisaje (VPAI), que expresa la calidad visual del paisaje en términos de conservación, refiriéndose al valor intrínseco (PIN) y el valor extrínseco (PEX). El valor máximo de los dos valores (PIN y PEX) se representa 0,75 y 0,25 puntos como se expresa en la siguiente Ecuación 1:

Ecuación 1: $\mathrm{VPAI}=0,75 \mathrm{PIN}+0,25$ PEX

En ese sentido, el Paisaje Intrínseco (PIN): Es la percepción que de cada unidad territorial o paisajística obtiene un observador situado en cualquier punto del entorno. Se compone de cinco características visuales: Fisiografía (FI), agua (AG), vegetación (VG), elementos artificiales (EA) y composición (CM). Cada factor se ponderó mediante un Delphi con el Grupo de expertos locales, según la Ecuación 2.

Ecuación 2: PIN $=0,1 \mathrm{FI}+0,1 \mathrm{AG}+0,2$ $\mathrm{VG}+0,2 \mathrm{EA}+0,4 \mathrm{CM}$

El Valor de la composición (CM): 
Anzaldúa-Soulé, Karla Rosalba; Almazán-Adame, Arely Adriana; Lorenzana Núñez, Oswaldo y Saldaña Almazán, Mirella

Potencial paisajistico de la Laguna de Coyuca de Benitez: Detonante de productos sustentables en Acapulco-México

Resulta de la combinación de distintos elementos visuales que conforman el medio físico, biótico y humano. Este valor surge de la agregación de dos componentes: Interacción (i) y cromatismo (c). El peso que se le otorga a cada uno es 0,6 y 0,4 como se expresa en la Ecuación 3.

Ecuación 3: $\mathrm{CM}=0,6 \mathrm{i}+0,4 \mathrm{c}$

$\mathrm{Al}$ respecto, la interacción (i) está definida por el grado de complejidad (cp) o un número de elementos (vegetación, relieve, agua), y el grado de armonía o naturalidad (ar) que determinan el paisaje. En ese sentido, el valor de la interacción se obtiene a partir de la suma de esos dos factores, se le otorga un peso mayor al factor armonía, puesto que la naturalidad y concordancia, son elementos que configuran el paisaje y al fusionarse otorgan al paisaje características intrínsecas. Los valores se pueden observar en la Ecuación 4:

Ecuación 4: i = 0,3 cp + 0,7 ar

Finalmente, en la Cuadro 2 se presenta el resumen de componentes, criterios y calificaciones para la valoración del paisaje intrínseco.

\section{Cuadro 2}

\section{Resumen para la valoración del paisaje intrínseco}

\begin{tabular}{|c|c|c|}
\hline Componentes & Criterios & Calificación \\
\hline \multirow{8}{*}{ Fisiografía } & Formas: & \\
\hline & Sobresaliente & 2 \\
\hline & Rasgos obvios pero no resaltan & 1 \\
\hline & Sin rasgos sobresalientes & 0 \\
\hline & Desarrollo vertical: & \\
\hline & Salientes & 3 \\
\hline & Llano & 2 \\
\hline & Abruptos & 1 \\
\hline \multirow{5}{*}{ Agua } & Laguna & 5 \\
\hline & Estero & 4 \\
\hline & Agua dulce y salada & 3 \\
\hline & Humedal & 2 \\
\hline & Sin presencia de agua & 0 \\
\hline \multirow{5}{*}{ Vegetación } & Selva baja caducifolia & 5 \\
\hline & Ecológica, florística y fisonómica & 4 \\
\hline & Agrícola, pecuaria y forestal & 3 \\
\hline & Asentamientos Humanos y zonas urbanas & 2 \\
\hline & Suelo desnudo & 1 \\
\hline \multirow{6}{*}{$\begin{array}{l}\text { Elementos } \\
\text { Artificiales }\end{array}$} & Ausencia de elementos artificiales & 5 \\
\hline & Intervención acorde con la aptitud del suelo e integrada al paisaje & 4 \\
\hline & $\begin{array}{l}\text { Intervención acorde con la aptitud del suelo en } 70 \% \text { integrada al } \\
\text { paisaje }\end{array}$ & 3 \\
\hline & $\begin{array}{l}\text { Intervención acorde con la aptitud del suelo en } 50 \% \text { integrada al } \\
\text { paisaie }\end{array}$ & 2 \\
\hline & $\begin{array}{l}\text { Intervención acorde con la aptitud del suelo en } 30 \% \text { integrada al } \\
\text { paisaje }\end{array}$ & 1 \\
\hline & Intervención no acorde con aptitud del suelo & 0 \\
\hline \multirow{12}{*}{ Composición } & Interacción $(0,3)$ : & Complejidad/ armonía \\
\hline & Muy alta & 5 \\
\hline & Alta & 4 \\
\hline & Media & 3 \\
\hline & Baja & 2 \\
\hline & Muy baja & 1 \\
\hline & Cromatismo $(0,7)$ : & $\begin{array}{c}\text { Diversidad, } \\
\text { variabilidad } \\
\text { estacional y contraste }\end{array}$ \\
\hline & Muy alta & 5 \\
\hline & Alta & 4 \\
\hline & Media & 3 \\
\hline & Baja & 2 \\
\hline & Muy baja & 1 \\
\hline
\end{tabular}

Fuente: Elaboración propia, 2020, a partir de Alberruche (2002); y, Delgado y Pantoja (2016). 
Por su parte, el Valor Extrínseco (PEX): Es la percepción que obtiene el observador en una determinada unidad de paisaje, del entorno que lo rodea. Este apartado identifica el potencial de atracción y expresa en función de la amplitud y profundidad del campo de visión (Pr), la calidad del tema de las visitas que percibe $(\mathrm{Ct})$ y la posición altitudinal $(\mathrm{PO})$ relativa, en la que se encuentra el observador, tal como se observa en la Ecuación 5:

Ecuación 5: $\mathrm{PEX}=0,4 \mathrm{Pr}+0,4 \mathrm{Ct}+0,2$ PO

En este sentido, en el Cuadro 3 se presenta un resumen de los componentes, criterios y calificación utilizados para la valoración del PEX.

\section{Cuadro 3}

\section{Resumen para la valoración del paisaje extrínseco PEX}

\begin{tabular}{lll}
\hline \multirow{2}{*}{ Componentes } & \multicolumn{1}{c}{ Criterios } & Calificación \\
\hline \multirow{4}{*}{ Profundidad visual } & Lejana & 5 \\
\cline { 2 - 3 } & Media alta distancia & 4 \\
\cline { 2 - 3 } & Media distancia & 3 \\
\cline { 2 - 3 } & Próximas & 2 \\
\cline { 2 - 3 } Calidad del tema & Inmediatas & 5 \\
\hline & Excelente & 4 \\
\cline { 2 - 3 } & Buena & 3 \\
\cline { 2 - 3 } & Regular & 2 \\
\cline { 2 - 3 } & Mala & 1 \\
\cline { 2 - 3 } Posición altitudinal & Muy mala & 3 \\
\hline & Posición superior & 3 \\
\cline { 2 - 3 } & A nivel & 1 \\
\cline { 2 - 3 } & A nivel más bajo & 3 \\
\hline
\end{tabular}

Fuente: Elaboración propia, 2020, a partir de Alberruche (2002); y, Delgado y Pantoja (2016).

De igual manera, el método para la valoración de la calidad del paisaje se realizó con el recorrido de campo a la zona; posteriormente, el equipo de trabajo desagregó los componentes, obteniendo los resultado VPIN y VPEX. Con la aplicación de las cuatro fases, se determinó el potencial paisajístico que pueden integrarse como una nueva propuesta de desarrollo turístico sustentable para Acapulco-México, promoviendo la diversificación de su producto turístico, que por décadas se ha apostado por impulsar el turismo de sol y playa, reconocido como un turismo de masas.

\section{Resultados y discusión}

\subsection{Condiciones socioeconómicas y ambientales de la Laguna de Coyuca de Benítez}

Se identificó en las condiciones socioeconómicas, áreas de poblaciones que habitan en los límites de la Laguna, seis localidades rurales vírgenes: La Barra de 
Anzaldúa-Soulé, Karla Rosalba; Almazán-Adame, Arely Adriana; Lorenzana Núñez, Oswaldo y Saldaña Almazán, Mirella

Potencial paisajístico de la Laguna de Coyuca de Benitez: Detonante de productos sustentables en Acapulco-México

Coyuca, los Mogotes, la Isla Montosa, el Embarcadero, Luces del Mar, San Nicolás de las Playas y una localidad urbana: Acapulco de Juárez; de dicha localidad se especificaron los espacios geográficos que colindan con la Laguna: Colonia Fuerza Aérea, Zona turística y centro del pueblo de Pie de la Cuesta.

Tal como se muestra en la Tabla 1, el total de población que habita los alrededores de la Laguna de Coyuca de Benítez, es de 14.335 habitantes, con una población indígena de 363, un total de viviendas de 3.869. En cuanto a los aspectos económicos, existe un total de 3.827 personas ocupadas, las cuales representan trabajadores y obreros eventuales o de planta; y en relación al personal desocupado, se obtuvieron 54 personas (que están en busca de activarse o tuvieron una ocupación previa) (INEGI, 2016).

Tabla 1

Análisis de condiciones socioeconómicas

\begin{tabular}{|c|c|c|c|c|c|c|}
\hline Descripción & $\begin{array}{l}\text { Población } \\
\text { total }\end{array}$ & $\begin{array}{l}\text { Población } \\
\text { Indígena }\end{array}$ & $\begin{array}{l}\text { Viviendas } \\
\text { habitadas }\end{array}$ & $\begin{array}{l}\text { Población } \\
\text { ocupada }\end{array}$ & $\begin{array}{l}\text { Población } \\
\text { desocupada }\end{array}$ & $\begin{array}{c}\text { Índice de } \\
\text { desarrollo } \\
\text { social }\end{array}$ \\
\hline \multicolumn{7}{|c|}{ Localidades Rurales } \\
\hline El Embarcadero & 1.370 & 15 & 370 & 576 & 0 & Muy bajo \\
\hline Luces del Mar & 1.200 & 29 & 309 & 514 & 12 & Bajo \\
\hline Los Mogotes & 1.284 & 38 & 336 & 525 & 9 & Bajo \\
\hline \multirow{3}{*}{$\begin{array}{l}\text { La Barra } \\
\text { San Nicolás de las } \\
\text { Playas } \\
\text { La Isla Montosa } \\
\text { (Área turística) }\end{array}$} & 907 & 10 & 247 & 417 & 6 & Bajo \\
\hline & 253 & 0 & 83 & 100 & 3 & $\mathrm{n} / \mathrm{d}$ \\
\hline & 5 & 0 & 3 & 3 & 0 & Medio \\
\hline \multicolumn{6}{|c|}{ Localidad urbana Acapulco de Juárez } & $\begin{array}{c}\text { Grado de } \\
\text { marginación }\end{array}$ \\
\hline $\begin{array}{l}\text { Colonia Fuerza } \\
\text { Aérea }\end{array}$ & 1.643 & 72 & 463 & 658 & 0 & $\begin{array}{c}\text { marginacion } \\
\text { Muy alto }\end{array}$ \\
\hline $\begin{array}{l}\text { Zona Turística Pie } \\
\text { de la Cuesta }\end{array}$ & 1.446 & 49 & 400 & 578 & 0 & Medio \\
\hline $\begin{array}{l}\text { Centro del } \\
\text { poblado Pie de la } \\
\text { Cuesta }\end{array}$ & 1.208 & 58 & 310 & 456 & 24 & Alto \\
\hline Total & 14.335 & 363 & 3.869 & 3.827 & 54 & \\
\hline
\end{tabular}

Fuente: Elaboración propia, 2020, adaptado del INEGI (2015b).

El índice de desarrollo social del 2010, en las localidades rurales como el Embarcadero, es muy alto, en tres localidades es bajo (Luces del mar, Los Mogotes y la Barra), y en la Isla Montosa es medio (ver Tabla 1). El grado de marginación, que obtuvieron las zonas colindantes a la Laguna de Coyuca de Benítez pertenecientes a la localidad urbana Acapulco de Juárez son: La colonia Fuerza Aérea, un grado muy alto; la zona turística Pie de la
Cuesta, fue medio; y el centro del poblado de Pie de la Cuesta, es alto.

Lo que se observó, es que en las áreas donde se desarrolla el turismo son las zonas que presentan menos niveles de rezago social y marginación como: La Isla Montosa y la Zona Turística de Pie de la Cuesta, es decir, presentan menos carencias sociales como rezago educativo, acceso a los servicios de salud, a los servicios básicos de vivienda y a la 
calidad de espacios en la vivienda, aun cuando el Estado de Guerrero presentó el mayor rezago social a nivel nacional (Consejo Nacional de Evaluación de la Política de Desarrollo Social [CONEVAL], 2018).

En cuanto a las condiciones ambientales, se identificó que: La Laguna de Coyuca, se localiza en la región hidrológica Costa Grande, es un cuerpo de agua perenne; además, los río de Coyuca, las Cruces y el Conchero, proporcionan materia orgánica, sales disueltas y sedimentos, que contribuyen al azolvamiento paulatino de la Laguna (Aguirre, 2001).

La provincia fisiográfica es la Sierra Madre del Sur; el tipo de clima es cálido subhúmedo; y los tipos de suelo que rodean la Laguna son: En mayor cobertura phaeozem, seguido de solonchak, arenosol y en menor extensión leptosol (INEGI, 2016).

Existen puntos diversos que colindan con la Laguna y se clasifican de acuerdo al desarrollo del territorio: El área que cubre mayor espacio es la clasificación agrícola, pecuaria, forestal; seguida de la categorización ecológica, florística y fisonómica; continuando con la clasificación de asentamientos humanos, zonas urbanas, que son áreas desprovistas de vegetación. El tipo de vegetación sobresaliente fue: Selva baja caducifolia, presente en la Isla Montosa, sin embargo, está siendo afectada por factores antrópicos; y en el área de manglar, que no ha sido afectada por factores antrópicos o naturales (INEGI, 2016).

\subsection{Valoración comunitaria participativa del paisaje de la Laguna de Coyuca de Benítez}

Con la aplicación de los talleres participativos, se identificaron los recursos tangibles e intangibles que integran los paisajes (naturales-ecológicos, históricos, estéticos, sociales, productivos, espirituales, simbólicos e identitarios). Asimismo, algunos hallazgos de los talleres fueron: En la primera parte, se identificaron los sitios donde se localizan los paisajes más atractivos y originales de la Laguna de Coyuca de Benítez, como: Las zonas de manglares, la Isla Pelona o de los Pájaros, la Barra, las huertas de cocos, la Isla Montosa, el faro, la puesta de sol, el Estero, la zona de restaurantes y hoteles, así como el Embarcadero. En la segunda parte, la población seleccionó cinco paisajes de los cuales se sienten orgullosos: La Isla Pelona o de los Pájaros, el Estero, la Barra, las zonas de manglares y la Isla Montosa.

Por último, los participantes realizaron un análisis de la realidad actual y del futuro: Los paisajes con mayores amenazas por los fenómenos naturales (ciclones), sobreexplotación de recursos naturales (madera, pesca, extracción de arena, caza de aves), vertedero de aguas residuales y presencia de residuos sólidos, realización de actividades sin regulación son, la Isla Pelona o de los Pájaros, el Estero, la Barra, las zonas de manglares y la Isla Montosa. En cuanto a su visión a futuro, manifestaron su preocupación por la pérdida de sus recursos, y propusieron generar brigadas y una agenda comunitaria, con el fin de comenzar a desarrollar acciones que beneficien su entorno.

Coincidiendo con la Organización de las Naciones Unidas para la Alimentación y la Agricultura (FAO, 2008), el contextualizar la realidad que se vive en la zonas identificadas por medio de la participación de la comunidad, sin duda proporciona a los miembros la posibilidad de emprender acciones precisas. Asimismo, los actores locales cuando se auto interpretan en sus realidades territoriales que se asocian, comunican, empoderan les permite influir y reflexionar sobre lo que ha de transformarse o conservar dentro de una ética de sustentabilidad (Delgado, 2018; Quispe, Ayaviri y Maldonado, 2018).

\subsection{Valoración de las características del paisaje}

Las fichas de observación, permitieron que un grupo de expertos analizaran ocho características del paisaje, subdivididas en variables que fueron estratificadas de acuerdo a la ponderación aplicada a cada paisaje. 
Anzaldúa-Soulé, Karla Rosalba; Almazán-Adame, Arely Adriana; Lorenzana Núñez, Oswaldo y Saldaña Almazán, Mirella

Potencial paisajístico de la Laguna de Coyuca de Benitez: Detonante de productos sustentables en Acapulco-México

Se analizaron cinco sitios con potencial paisajístico de acuerdo a sus características: La Barra, con la mayor ponderación de 70; seguido de la Isla Montosa, con 69 puntos; el
Estero, con 62; la Isla Pelona, con 61; y, las zonas de manglares con 50 puntos, como se observa en la Tabla 2.

Tabla 2

Valoración por medio de la ponderación de fichas de observación

\begin{tabular}{lccccc}
\hline \multicolumn{1}{c}{ Descripción } & $\begin{array}{c}\text { Isla } \\
\text { Pelona }\end{array}$ & El Estero & $\begin{array}{c}\text { Zonas de } \\
\text { manglares }\end{array}$ & $\begin{array}{c}\text { Isla } \\
\text { Montosa }\end{array}$ & $\begin{array}{c}\text { La } \\
\text { Barra }\end{array}$ \\
\hline 1. Denominación del paisaje & 1 & 1 & 1 & 1 & 1 \\
2. Valoración del paisaje & 5 & 6 & 3 & 6 & 5 \\
3. Estado de conservación & 4 & 4 & 4 & 3 & 4 \\
4. Grado de vulnerabilidad & 1 & 1 & 1 & 1 & 1 \\
5. Valor patrimonial intrínseco del paisaje & 14 & 14 & 11 & 11 & 13 \\
desde el Punto de vista turístico & 15 & 13 & 11 & 14 & 14 \\
6. Atracción & 5 & 5 & 4 & 10 & 15 \\
7. Accesibilidad & 16 & 18 & 15 & 23 & 17 \\
8. Lugares de equipamiento & Total & 61 & 62 & 50 & 69 \\
\hline
\end{tabular}

Fuente: Elaboración propia, 2020, a partir de la ficha de observación de Anzaldúa-Soulé (2013).

Posteriormente, el análisis integral de las seis variables: Factores fisiográficos; usos y las cubiertas del suelo; dimensión histórica del paisaje; estructura del paisaje; visibilidad; dinámicas recientes y sentimientos del lugar; arrojaron la detección de cinco unidades de paisaje: La Barra, la isla Montosa, el Estero, la isla Pelona y la zona de manglares.

\subsection{Evaluación de calidad paisajística con potencial turístico}

Con base en la aplicación de la metodología de valoración mixta, para determinar la calidad paisajística se obtuvieron los siguientes sitios candidatos. En la Tabla 3, se encuentra la valoración numérica y el orden de prioridad de los mismos.

Tabla 3

Valoración paisajística de los sitios con potencial turístico de la Laguna de Coyuca de Benítez

\begin{tabular}{lccccccccccc}
\hline Sitios potenciales & FI & AG & VG & EA & CM & PIN & PR & CT & PO & PEX & VPAI \\
\hline 1. Isla Pelona & 0.40 & 0.50 & 0.80 & 0.80 & 1.91 & 3.31 & 1.00 & 1.20 & 1.00 & 0.80 & 4.11 \\
2. Isla Montosa & 0.40 & 0.50 & 1.00 & 0.40 & 1.69 & 2.99 & 1.00 & 1.20 & 1.00 & 0.80 & 3.79 \\
3. El Estero & 0.20 & 0.40 & 0.80 & 0.60 & 1.82 & 2.87 & 0.80 & 1.20 & 0.80 & 0.70 & 3.57 \\
4. Zona de manglares & 0.20 & 0.20 & 0.60 & 0.80 & 1.56 & 2.52 & 0.80 & 1.20 & 1.00 & 0.75 & 3.27 \\
5. La Barra & 0.30 & 0.30 & 0.40 & 0.20 & 1.39 & 1.94 & 0.60 & 1.00 & 0.80 & 0.60 & 2.54
\end{tabular}

Fuente: Elaboración propia, 2020 con resultados de la aplicación de la metodología adaptada de Alberruche (2002); y, Delgado y Pantoja (2016). 


\subsection{Análisis de la valoración del paisaje intrínseco (PIN) de los sitios de la Laguna de Coyuca de Benítez}

\section{a. Valor de la Fisiografía (FI)}

La fisiografía del territorio analizado es parte de la Sierra Madre de Sur, que se extiende a lo largo y cerca de la costa del pacífico de Guerrero. Se encuentra dentro de la provincia Balsas Sudpacifiquense, y morfométricamente se clasifica como tipo tres (Lankford, 1977). El territorio de la Laguna de Coyuca de Benítez, es un relieve costero, donde sobresalen de acuerdo a sus características las dos islas Pelona y Montosa, seguida de las zonas llanas el Estero, zona de manglares y la Barra.

\section{b. Agua (Ag)}

La ponderación con mayor valor fue el cuerpo de agua Laguna, área que rodea las dos islas Pelona y Montosa, seguida del Estero y la zona de manglares, donde desemboca el Río de Coyuca que se une con la Laguna de Coyuca de Benítez, por último, la Barra es una bifurcación natural entre la Laguna de agua dulce y el Océano Pacífico.

\section{c. Vegetación (VG)}

Con el análisis se observa que el territorio presenta un significativo grado de intervención antrópica especialmente en lo que respecta a la vegetación, de manera que el único lugar denominado selva baja caducifolia, es la Isla Montosa, aun cuando su superficie es 154.880 metros cuadrados, presenta un espacio antropizado de aproximadamente 11.000 metros cuadrados, principalmente por el desarrollo de actividades turísticas.

La valoración del paisaje intrínseco de acuerdo con la calificación especificada en la Tabla 2, se obtuvo la designación de cuatro, denominada por sus características agrícolas, pecuaria y forestal, en la cual se consideraron:
La Isla Pelona o de los Pájaros y el Estero; la isla, tiene una extensión de 42.440 metros, principalmente su uso es escénico puesto que los recorridos turísticos en la zona aprovechan su calidad ambiental para actividades de observación de flora y fauna. En lo que respecta al Estero, funciona como bebedero natural del ganado que habita en el territorio colindante, y en las zonas más bajas de profundidad, funciona como área de recreación turística.

El área con valoración tres, fue la zona de manglares, denominada ecológica, florística y fisonómica, esta área solo se utiliza para actividades turísticas de observación, por sus características naturales y su valor escénico. Por último, el valor dos, con denominación de asentamientos humanos, la Barra, en ella se localizan diversas construcciones de viviendas y negocios, mismo que han modificado las condiciones del suelo.

\section{d. Elementos artifíciales (EA)}

En el territorio de la Laguna de Coyuca de Benítez, las zonas con ponderación más alta por la ausencia de elementos artificiales son: El Estero, la Isla Pelona y la zona de manglares; y las zonas con valor de dos, es la Barra y la Isla Montosa, puesto que, presentan una intervención de aptitud del suelo del $30 \%$ integrada al paisaje, principalmente, esto se debe a actividades agrícolas, pesqueras y la de mayor presencia turística.

\section{e. Composición (CM)}

En cuanto a la complejidad, la Isla Pelona, el Estero y la zona de manglar, presentan una calificación de 5 (ver Tabla 2); con relación a la armonía, la Barra y la Isla Montosa, a pesar de tener mayor intervención por el hombre, hacen que los visitantes logren explorar el área, conociendo su historia, sus leyendas e interactúen con la población local. En lo que respecta a la valoración del cromatismo, el Estero y la Isla pelona tienen una especial importancia por sus aspectos 
Anzaldúa-Soulé, Karla Rosalba; Almazán-Adame, Arely Adriana; Lorenzana Núñez, Oswaldo y Saldaña Almazán, Mirella

Potencial paisajistico de la Laguna de Coyuca de Benitez: Detonante de productos sustentables en Acapulco-México

físicos y por sus matices que contrastan con colores: Verdes, azules y naranjas.

\section{f. Valor total intrínseco (PIN)}

Se recuerda que el PIN representa el $75 \%$ del total de la valoración del paisaje y el $25 \%$ restante se completa con el PEX que se detalla más adelante. De la suma de todos los elementos evaluados, al aplicar la Ecuación 2 se obtiene el PIN. La mayor calificación fue 3,31 siendo obtenida por la Isla Pelona.

\subsection{Valor del paisaje extrínseco (PEX)}

\section{a. Profundidad visual (PR)}

No se obtuvieron ponderaciones de visualizaciones lejanas y media alta distancia, la categoría mayor fue la de media distancia asignadas a: La Isla Pelona e Isla Montosa, coincidiendo por su mayor altitud, seguida de una denominación visual próxima, para el caso del Estero y la zona de manglar; y por último, como visual inmediata, la Barra.

\section{b. Calidad del tema (CT)}

Ninguno de los sitios obtuvo una calificación mala o muy mala, un sitio se encuentra en excelente, tres en buena y solo la Barra, aparece con una asignación regular, analizando que es la que presenta mayor antropización.

\section{c. Posición altitudinal (PO)}

No se obtuvo sitios con valor uno de posición superior, la mayoría se concentró en cinco y tres. La calificación de cinco, la obtuvieron los sitios que presentan mayor altitud como la zona de manglares, las islas Pelona y Montosa; seguida de las zonas llanas como el Estero y la Barra.

\section{d. El total del PEX}

Al aplicar la Ecuación 4, se obtuvo los sitios con mayor calificación: La Isla Pelona y la Isla Montosa.

\section{e. Valor total de la calidad visual del paisaje (VPAI)}

Al unir los valores PIN + PEX para obtener el VPAI, conforme a la Ecuación 1, los mayores valores fueron (ver Gráfico I): En primer nivel, la Isla Pelona; y con resultados equilibrados, la Isla Montosa, el Estero y la zona de manglares. 


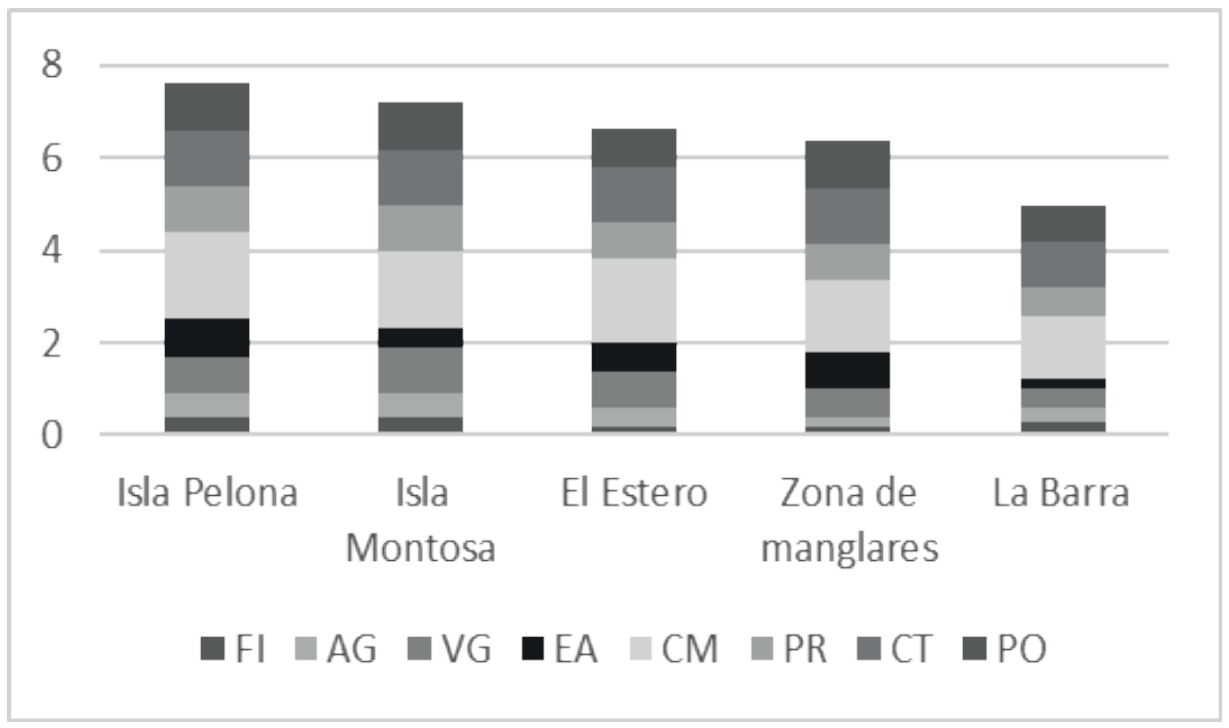

Fuente: Elaboración propia, 2020.

\section{Gráfico I: Valoración de la calidad visual de paisajes PIN + PEX}

\subsection{Unidades de paisaje}

Las unidades de paisaje, se estructuraron con base en los resultados de la investigación y la aplicación de la metodología propuesta por Nogué y Sala, (2008). Por lo tanto, de acuerdo a las características propias de cada sitio se generaron cinco unidades, subdivididas en paisaje rural, natural y urbano. Como rural, se integró el Estero; como natural: La Isla la pelona y la zona de manglares; y urbano: La Barra y la Isla Montosa; aun cuando esta isla todavía cuenta con una amplia extensión territorial sin modificaciones por el hombre, es un área que constantemente se encuentra aprovechada por las actividades turísticas.

Por otra parte, las leyes y reglamentos de los municipios de Coyuca de Benítez y Acapulco de Juárez, presentan vacíos sobre la protección, ordenación y gestión del paisaje, por lo tanto, se necesita generar instrumentos para establecer medidas, procedimientos en el cuidado y aprovechamiento del paisaje. Las sinergias en el desarrollo de las nuevas investigaciones del paisaje, deberán tener una visión de protección de los valores ambientales, históricos-culturales, pretendiendo desarrollar actividades que no produzcan una variación en sus recursos turísticos.

Algunas metodologías que han sido aplicadas con éxito en el análisis del paisaje desde una perspectiva sustentable y el potencial turístico son: La valoración del paisaje, en una propuesta de turismo sostenible: la "Ruta del Oro', Nariño (Colombia) (Delgado y Pantoja, 2016); la valoración paisajística del potencial turístico de la reserva del Río Playa de Comalcalco, Tabasco (Liévano, Juárez y Mazo, 2014); y la valoración del paisaje y evaluación del potencial interpretativo o como herramienta para el turismo sostenible en el Ecoparque Las Monjas (La Mesa, Cundinamarca) (Reyes, et al., 2017).

Sin embargo, de acuerdo al alto valor 
paisajístico identificado en las unidades de paisaje, es necesario seguir trabajando en el monitoreo sobre el desarrollo de las actividades turísticas bajo la óptica de la planeación sustentable, que permita no demeritar el recurso paisajístico y preservarlo para las generaciones futuras, tratando de no cometer los mismos errores que se han producido en anteriores paisajes del destino turístico Acapulco. Concretizando, que la suma de la aplicación del turismo sustentable, ético y seguro, debe de ser valorado por los turistas, gobernantes, empresarios y la comunidad (Santamaría-Freire y López-Pérez, 2019).

Coincidiendo con Gutiérrez y Narváez (2015), el desarrollo competitivo del turismo, se fundamenta en su capacidad para generar prosperidad económica sostenida, incluyendo aspectos como la calidad de vida de los ciudadanos y la sustentabilidad ambiental, sobre la base del uso de los atractivos naturales, económicos y sociales.

\section{Conclusión}

El paisaje de la Laguna de Coyuca de Benítez, es un elemento integrador de recursos naturales y culturales, siendo de gran importancia para la actividad turística del destino turístico Acapulco-México. Además, el paisaje de la zona presenta calidad visual, que no puede separarse de una correcta ordenación enfocada en el desarrollo sostenible de un territorio. Sin embargo, los paisajes degradados evidencian una inconciencia social sobre la gestión y se considera una baja apreciación emocional y afectiva de la comunidad con el entorno.

Es por ello, que se debe establecer un proceso continuo de evaluación enmarcado por objetivos que buscan la calidad paisajística, por lo tanto, requieren un seguimiento constante, con la finalidad de introducir las medidas preventivas o correctivas que resulten necesarias en cualquier actividad que se desarrolle en el territorio.

Por tal motivo, las nuevas actividades turísticas que se deslindan a partir de la calidad visual, deben ser soportables ecológicamente a largo plazo, viables económicamente y equitativas desde una perspectiva ética así como social para las comunidades locales.

Precisando, las cinco unidades de paisaje con alta calidad visual de la Laguna de Coyuca de Benítez, podrán representar una motivación importante de desplazamiento de turistas, sin embargo, durante el aprovechamiento se debe prever la diversificación de actividades económicas, al tiempo que garantice el menor impacto negativo de las tradiciones, la cultura y el medio ambiente.

Finalmente, se debe generar una conciencia paisajística en la población y en el turista, debido a que es una condición para que se realice un uso adecuado del paisaje y cooperen con la protección de los recursos. Puesto que, cualquier medida de ordenación, protección y gestión del paisaje, será insuficiente y en algunos casos extremos, imposibles de conseguir sin la participación de la sociedad.

\section{Referencias bibliográficas}

Aguilar, A., Palafox, A., y Anaya, J. S. (2015). El turismo y la transformación del paisaje natural. Nóesis. Revista de Ciencias Sociales y Humanidades, 24(47-1), 19-29.

Aguirre, R. (2001). Caracterización óptica de la laguna costera de Coyuca de Benítez. Investigaciones Geográficas, (46), 78-97.

Alberruche, E. (2002). El análisis de la fragilidad visual mediante Sistemas de Información Geográfica. En L. Laín (Ed.), Los sistemas de información geográfica en la gestión de los riesgos geológicos y el medio ambiente. Instituto Geológico y Minero de España (IGME).

Alcaraz, O., y Salgado, A. C. (2019). El paisaje patrimonial para la promoción turística. Acapulco y Taxco, Guerrero. 
Revista de Arquitectura, Urbanismo y Territorios, 1(17), 178-198.

Anzaldúa-Soulé, K. (2013). El aprovechamiento turístico del paisaje desde una visión sustentable: Una propuesta para Pie de la Cuesta, Municipio de Acapulco (Tesis de maestría). Instituto Politécnico Nacional, Ciudad de México, México.

Boerema, A., Rebelo, A. J., Body, M. B., Esler, K. J., y Meire, P. (2017). Are ecosystem services adequately quantified? Journal of Applied Ecology, 54(2), 358-370. https://doi. org/10.1111/1365-2664.12696

Bramwell, B., y Lane, B. (1993). Interpretation and sustainable tourism: The potential and the pitfalls. Journal of Sustainable Tourism, 1(2), 71-80.

Burkhard, B., Kroll, F., Muller, F., y Windhorst, W. (2009). Landscapes“ capacities to provide ecosystem services - A concept for land-cover based assessments. Landscape Online, 15, 1-22. https://doi.org/10.3097/ $\underline{\mathrm{LO} .200915}$

Consejo Nacional de Evaluación de la Política de Desarrollo Social - CONEVAL (2018). Medición de la Pobreza. https://www.coneval.org.mx/ Medicion/Paginas/PobrezaInicio.aspx

Delgado, J. C. (2018). Dimensión prospectiva del actor local en el talento territorial. Revista de Ciencias Sociales (Ve), XXIV(2), 83-93.

Delgado, A. M., y Pantoja, F. (2016). Valoración del paisaje en una propuesta de turismo sostenible: La "Ruta del Oro", Nariño (Colombia). Cuadernos de Geografia: Revista Colombiana de Geografia, 25(1), 233-253.

Dos Santos, P. (2011). Marco teóricometodológico de los estudios del paisaje. Perspectivas de aplicación en la planificación del turismo. Estudios y Perspectivas en Turismo, 20(3), 522541.

Guillén, J., Calle, J., Gavidia, A. M., y Vélez, A. G. (2020). Desarrollo sostenible: Desde la mirada de preservación del medio ambiente colombiano. Revista de Ciencias Sociales (Ve), XXVI(4), 293-307. https://doi.org/10.31876/rcs. v26i4.34664

Gutiérrez, M., y Narváez, M. (2015). Lineamientos estratégicos para el desarrollo competitivo del sector turísticos en Venezuela. Revista de Ciencias Sociales (Ve), XXI(3), 416428.

Hernández, R., Fernández, C., y Baptista, P. (2014). Metodología de la investigación. McGraw-Hill.

Instituto Nacional de Estadística y Geografía - INEGI (2015a). Catálogo del Territorio Insular Mexicano. INEGI. https://bit.ly/3v1hw9W

Instituto Nacional de Estadística Geografía - INEGI (2015b). Sistema para la Consulta de Información Censal. INEGI. http://gaia.inegi.org.mx/ scince2/viewer.html

Instituto Nacional de Estadística y Geografía - INEGI (2016). Mapa Digital de México. https://bit.ly/3x96K3A

Lankford, R. R. (1977). Coastal Lagoons of Mexico, their origin and classification. In L. E. Cornin (Ed.), Estuarine Processes. Circulation, sediments and transfer of material in the estuary (pp. 182-215). Academic Press. https:// doi.org/10.1016/B978-0-12-751802$2.50022-9$

Liévano, K., Juárez, J. F., y Mazo, M. L. (2014). Valoración paisajística del potencial turístico de la reserva Río Playa de Comalcalco, Tabasco. Teoría y Praxis, 10(E), 138-157.

Macbeth, J., Carson, D., y Northcote, J. K. 
Anzaldúa-Soulé, Karla Rosalba; Almazán-Adame, Arely Adriana; Lorenzana Núñez, Oswaldo y Saldaña Almazán, Mirella

Potencial paisajístico de la Laguna de Coyuca de Benitez: Detonante de productos sustentables en Acapulco-México

(2004). Social capital, tourism and regional development: SPCC as a basis for innovation and sustainability. Current Issues in Tourism, 7(6), 502522. https://doi.org/10.1080/13683500 $\underline{50408668200}$

Maloguelonne Déjeant-Pons Head of the Spatial Planning and Landscape Division (2006). The European Landscape Convention. Landscape Research, 31(4), 363-384. https://doi. org/10.1080/01426390601004343

Márquez-García, A. Z., Campos-Verduzco, R., y Castro-Soriano, B. S. (2010). Sedimentología y morfología de la playa de anidación para tortugas marinas, El Carrizal, Coyuca de Benítez, Guerrero. Hidrobiológica, 20(2), 101-112.

Méndez,A., García, A., Serrano, M.A., e Ibarra, V. (2016). Determinantes sociales de la viabilidad del turismo alternativo en Atlautla, una comunidad rural del Centro de México. Investigaciones Geográficas, (90), 119-134. https:// doi.org/10.14350/rig.48297

Méndez-Méndez, A., Serrano, M. A., Salinas, E., y García-Romero, A. (2018). Propuesta metodológica basada en indicadores para la valoración del potencial turístico del paisaje en áreas rurales: El caso del municipio de Atlautla (México). Cuadernos de Turismo, (42), 335-354. https://doi. org/10.6018/turismo.42.15

Narváez, M., Fernández, G., y Gutiérrez, C. (2018). Participación de las comunidades organizadas en el Desarrollo Turístico Local (ParaguanáVenezuela). Revista Venezolana de Gerencia, 23(81), 106-120.

Nogué, J. (1992). Turismo, percepción del paisaje y planificación del territorio. Estudios Turísticos, (115), 45-54.

Nogué, J., y Sala, P. (2008). El paisaje en la ordenación del territorio. Los catálogos de paisaje de Cataluña. Cuadernos Geográficos, (43), 69-98.

Núñez, J. M., Fuentes, F. J., y Sánchez, S. M. (2014). Actitudes del residente hacia el impacto del desarrollo turístico: estudio empírico. Revista Venezolana de Gerencia, 19(66), 209-227.

Ordoñez, A., y Ochoa, P. (2020). Ambiente, sociedad y turismo comunitario: La etnia Saraguro en Loja - Ecuador. Revista de Ciencias Sociales (Ve), XXVI(2), 180-191. https://doi. org/10.31876/rcs.v26i2.32433

Organización de las Naciones Unidas para la Alimentación y la Agricultura - FAO (2008). La FAO en México: Más de 60 años de cooperación 1945 - 2009. FAO. http://www.fao.org/3/be792s/ be792s.pdf

Pelegrín, N., Martínez, O., Pelegrín, A., y Zaballa, E. L. (2020). Senderismo como opción para pequeñas ciudades patrimoniales en regiones turísticas: Trinidad de Cuba. Revista de Ciencias Sociales (Ve), XXVI(3), 231243. https://doi.org/10.31876/rcs. v26i3.33244

Quispe, G., Ayaviri, D., y Maldonado, R. (2018). Participación de los actores en el desarrollo local en entornos rurales. Revista de Ciencias Sociales (Ve), $X X I V(3), 62-82$

Reyes, A. C., Torres, J. L., Villarraga, L. F., y Meza, M. C. (2017). Valoración del paisaje y evaluación del potencial interpretativo como herramienta para el turismo sostenible en el Ecoparque Las Monjas (La Mesa, Cundinamarca). Cuadernos de Geografia: Revista Colombiana de Geografía, 26(2), 177194. http://dx.doi.org/10.15446/rcdg. v26n2.61088

Santamaría-Freire, E. J., y López-Pérez, S. D. L. A. (2019). Beneficios social de la 
actividad turística en Ecuador. Revista Venezolana de Gerencia, 24(86), 417434.

Stoffelen, A., y Vanneste, D. (2015). An integrative geotourism approach: Bridging conflicts in tourism landscape research. Tourism Geographies. 17(4), 544-560. https://doi.org/10.1080/1461 $\underline{6688.2015 .1053973}$

Suárez, R. H. (2014). El paisaje como recurso turístico en las áreas naturales protegidas: caso reserva de la Biosfera Janos, Chihuahua, México. NovaRua, 4(9), 6-13. http://dx.doi.org/10.20983/ novarua.2014.9.1

Van den Berg, A. E., y Van Winsum-Westra, M. (2010). Manicured, romantic, or wild? The relation between need for structure and preferences for garden styles. Urban Forestry \& Urban Greening, 9(3), 179-186. https://doi.

\section{org/10.1016/j.ufug.2010.01.006}

Weyland, F., y Laterra, P. (2014). Recreation potential assessment at large spatial scales: A method based in the ecosystem services approach and landscape metrics. Ecological Indicators, 39, 34-43. https://doi. org/10.1016/j.ecolind.2013.11.023

Włodarczyk, B. (2009). The landscapes of tourism space. Tourism, 19(1-2), 8390. https://doi.org/10.2478/V10106009-0011-z

Wozniak, E., Kulczyk, S., y Derek, M. (2017). From intrinsic to service potential: An approach to asses tourism landscape potential. Landscape and Urban Planning, 170, 209-220. https://doi.org/10.1016/j. landurbplan.2017.10.006 